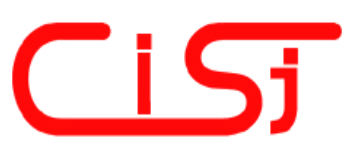

\title{
ALTERATION CORRECTION IN LYMPHOCYTE IMAGE FOR MICRO NUCLEUSES DETECTION
}

\author{
Domenico Luca Carnm, Domenico Grimaldi, Francesco Lamonaca \\ Dept. of Electronics, Computer and System Sciences, Univ. of Calabria, 87036 Rende -CS, Italy \\ Ph.:+39 0984 494712, fax: +390984 494713, \{dlcarni, grimaldi\}@deis.unical.it
}

\begin{abstract}
The paper presents a method pointed out to detect and to correct the alterations of (i) under-exposure, (ii) overexposure, (iii) out of focus, (iv) Gaussian noise, affecting the images acquired into the flow cytometer. These alterations reduce the image quality and interfere with the correct micro nucleus detection in lymphocyte.

The objective of the proposed correction is to make the image able to be correctly processed by the pattern matching algorithm (i) to detect the micronucleus into human lymphocytes, (ii) to minimize the doubtful detections, and (iii) to enhance the confidence that in the rejected images are not including the micro nucleuses.

The results of numerical tests confirm the validity of the proposed correction method.
\end{abstract}

Keywords: Fflow cytometer, pattern matching, image quality, under-exposure, over-exposure, out of focus, Gaussian noise.

\section{INTRODUCTION}

In order to relieve structural chromosome aberrations [1]-[3], recently in literature was presented a new architecture of flow cytometer [4] able to recognize and automatic count the micro nucleuses on the acquired images of human lymphocytes. The micronucleus detection is performed by means of pattern matching algorithm. Both the noise and the alteration affecting the acquisition system misstate the acquired image [5]-[7] and reduce the Image Quality (IQ). It can occurs that the micro nucleus can not be detected by the pattern matching algorithm in consequence of the image alteration and not for its effective absence. As a consequence, the number of the correct detection performed by the pattern matching algorithm can reduce and the number of both the doubtful and rejected detections increases. Important aspects for diagnosis are (i) the minimization of the doubtful detections, and (ii) the confidence enhancement that the rejected images are not including the micro nucleuses.

The problems arising from the image alteration can be conveniently clashed by the image pre-processing before the micronucleus detection by the pattern matching algorithm. The aim of the image preprocessing is (i) the evaluation of the IQ, (ii) the detection of the different alterations causing the bath IQ, and, consequently, (iii) the contrast and the correction of each alteration so as the IQ is enhanced.

In literature there are methods to a priori evaluation of (i) the indexes estimating the IQ, (ii) the type of the disturbances reducing the IQ, and (iii) the correction procedure of each type of the detected disturbances to improve the IQ [5]-[8]. In general, the image correction methods can be distinguished in [5]-[7] and [9]: (i) punctual, whether the new value of the pixel in exam depend on the value of the same pixel, (ii) local, whether the new value of the pixel in exam depend on the value of the pixels in his neighborhood, and (iii) global, whether the new value of the pixel in exam depends on the global image. Another type of sorting depends on the domain in witch the correction methods work: in the frequency or in the spatial domain.

On the basis of (i) the different characteristics of the acquired image from the flow cytometer, (ii) the type and the amplitude of the alterations, and (iii) the form of the border to be detected, not all the methods presented in literature are effective. Therefore, it needs the selection among all the methods in order to perform the correction and to obtain the desired results by the image processing.

The objective of the paper is to start from the methods available in literature (i) to point out, (ii) to adapt, (iii) to implement and (iv) to calibrate the image correction methods in order to increase the number of the correct detection of the micro nucleuses on the images of human lymphocytes.

In particular, the alterations taken into account in the paper are: (i) both the overexposure and the underexposure, (ii) the out of focus, and (iii) the Gaussian noise. Because the previous listed alterations influence in different way the pattern matching algorithms available in literature, the first step is to 
experiment and to select the pattern matching algorithm offering the best results regarding to the pre-processing performed on the acquired image. The successive steps deal with the paper are (i) the definition and the evaluation of indexes estimating the image alteration, (ii) the evaluation of the variation range of each index in order to permit the correction of the acquired image, and (iii) the chosen, the adaptation, the implementation and the test of the convenient correction method of each image alteration taken into account.

\section{CHOICE OF THE PATTERN MATCHING ALGORITHM}

Among the several pattern matching algorithms available in literature [10], that one implemented in the IMAQ Vision [11]-[13] is selected to be used in the new architecture of the flow cytometer [4] according to the following properties: (i) it is independent from the shape of the template to be detected, (ii) it is fast owing to the optimized learning phase, and (iii) it offers the best results regarding to the correction methods performed during the pre-processing phase. The calibration of the pattern matching algorithm has been carried out with the objective to reduce the sensitivity to the aforesaid alterations. That has been obtained with the following passages: (i) individuation of the images to be analyzed, (ii) study of the images by human operator in order to extract the number and the position of the micro nucleuses, (iii) analysis by the IMAQ tool by varying the calibration parameters, and (iv) comparison between the automatic and the human results. The chosen parameters are those that maximize the number of images correctly recognized, for which the result supplied from the tool is practically coincident with that one furnished by the operator.

\section{IMAGE PRE-PROCESSING TO PERFORM THE CORRECTION}

The general scheme to pre-process the image in order to correct the alteration is shown in Fig.1. The steps to be performed are:

a. Estimation index of the image alteration: three different indexes are carried out on the basis of the three different image alterations taken into account. In particular: (i) to detect bad exposure, the mean value of the grey levels is assumed as index, (ii) to detect out of focus, the mean value of the grey levels of the Prewit filter of image is assumed as index, and (iii) to detect Gaussian noise, the mean value of the minimum values of the grey levels obtained by the high pass filter applied by windowing the original image is assumed as index.

b. Evaluation of the index range to image correction: by referring to each of the previous evaluated indexes it is established if it needs to process the image and to correct the alteration. The decision is carried out on the basis of the index value compared with the admissible variation range. Than, it needs to evaluate both the upper and the lower values of this variation range according to the characteristics of the pattern matching algorithm.

c. Image correction: on the basis of the three different indexes, three different correction methods are pointed out. The correction depends on (i) the disturbance type, and (ii) the value of the corresponding index.

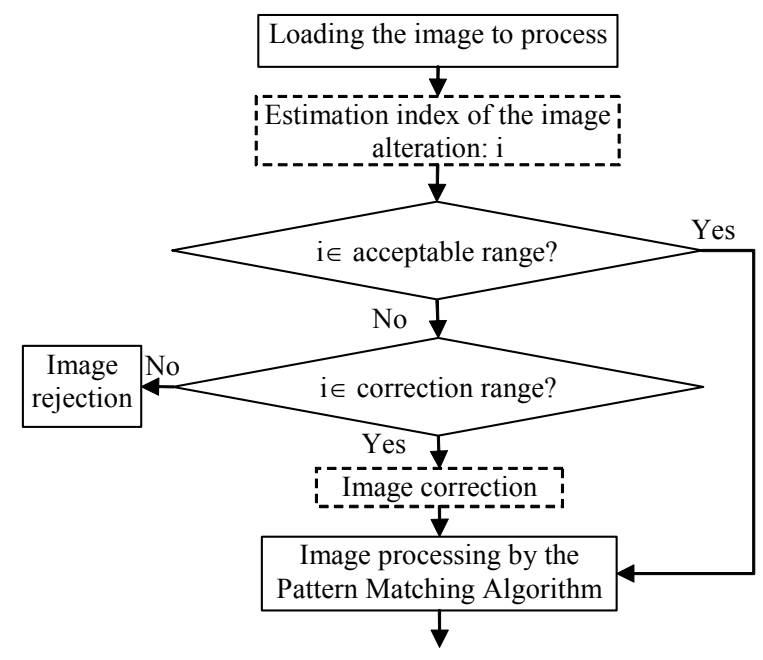

Fig.1 - Block scheme (i) to estimate the index of the image alteration, and (ii) to correct the image.

The dashed blocks of Fig.1 are particularized according to the image alteration under examination. Once pre-processed and corrected according to the previous block scheme, the resulting image is able to be analyzed by the pattern matching algorithm and, consequently, the response is characterized by high level of confidence.

\section{OVEREXPOSED AND UNDEREXPOSED ALTERATION}

In order to evaluate the overexposure and underexposure the valid index is evaluated by taking into account the image histogram.

Two types of image histograms can be distinguished: linear and cumulative. In the following the linear histogram is used. This histogram is the function $\mathrm{H}$ defined on the grey scale ranging in the interval $[0,255]$ so as the number of pixels characterized by the same grey-level $\mathrm{k}$ is $\mathrm{H}(\mathrm{k})=\mathrm{n}_{\mathrm{k}}$ [11].

The histogram of the overexposed image is concentrated into the bright region, and in the dark region for the underexposed image, as shown in Fig.2.

From the histogram the following indexes can be gained: (i) mean value, (ii) standard deviation, (iii) maximum value, (iv) minimum value, (v) range of grey 
level. After numerical tests, the convenient index for the bad exposure examination is the mean value.

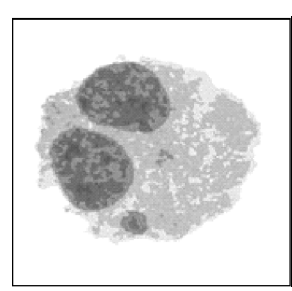

$\mathbf{a}$

c

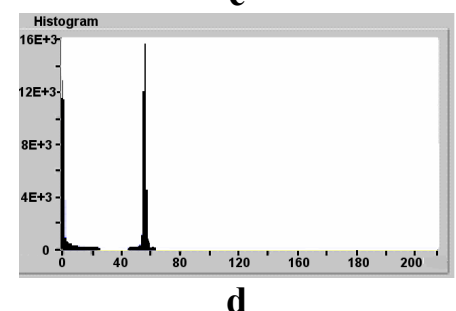

d

b

\section{.}
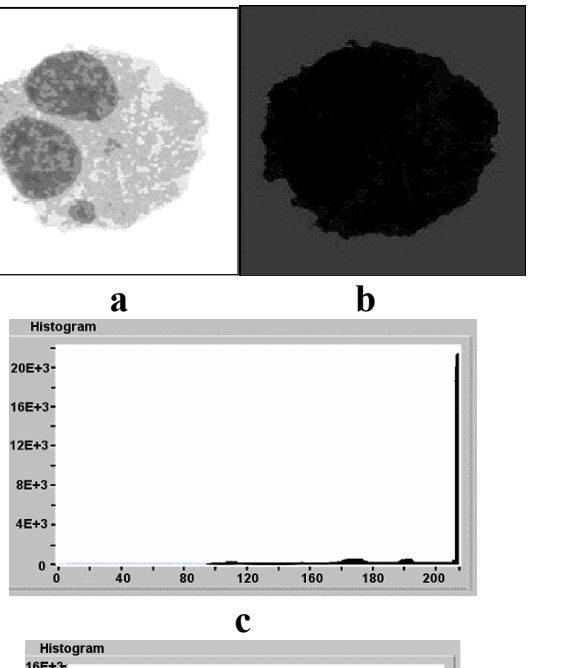

Fig.2 - Exposure alteration a) overexposed image and c) corresponding histogram; b) underexposed image, and d) corresponding histogram.

To evidence the presence of the micro nucleuses into the overexposed image the empiric method is pointed out. Numerical tests show that adding 255 to the underexposed images or -255 to overexposed images the presence of the micro nucleuses can be evidenced. In particular, by applying the method to the overexposed images of Fig.3a, the obtained image is scattered and is shown in Fig.3b. The micro nucleus is not detectable directly. Solution to this problems consists in (i) inverting the image (Fig.3c), and (ii) filtering by means of the Gaussian filter with standard deviation equals to 0,625 . This filter maximizes the number of degraded image correctly analyzed. Fig.3d shows the final image that is correctly evaluated by the pattern matching algorithm.

Fig. 4 shows the block scheme to detect and to correct the bad exposition of the image. The image is loaded in memory and the mean value of the grey level is estimated. After the tests with the threshold value, the source image is passed into (i) the histogram translator block, (ii) the convolution block, and (iii) the inversion block to determine the image that can be passed to the pattern matching algorithm. If the value of the bad exposure index is bigger than 252,10 the image must be considered overexposed, if lower than 53,00 the image must be considered underexposed. In both the cases the image needs to be corrected. The correction can be conveniently performed if the index is not greater than the value 254,70 and not lower of 35,17 . Out of this range the image must be rejected. These values was found by means of numerical tests. The value 252,10 is the bad exposure index of the last image correctly evaluated by the pattern matching algorithm without correction. The value 254,70 is the bad exposure index of the last image correctly evaluated by the pattern matching algorithm after the proposed correction. In similar way was found both the values 53,00 and 35,17. They are the bad exposure indexes corresponding to the last image correctly evaluated before and after the use of the proposed correction.

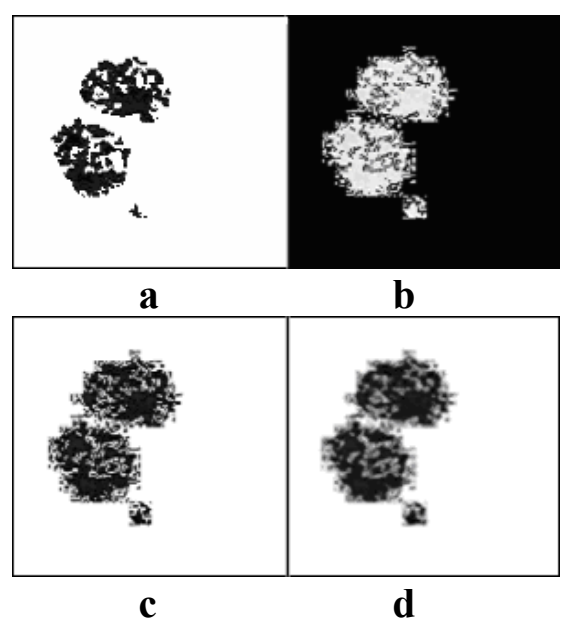

Fig.3 - a) Overexposed image to be corrected, b) previous image obtained by summing $\mathbf{- 2 5 5}$, c) inversion of the image $b$ ), and d) image c) filtered by Gaussian filter with $\mathbf{y}=\mathbf{0 , 6 2 5}$.

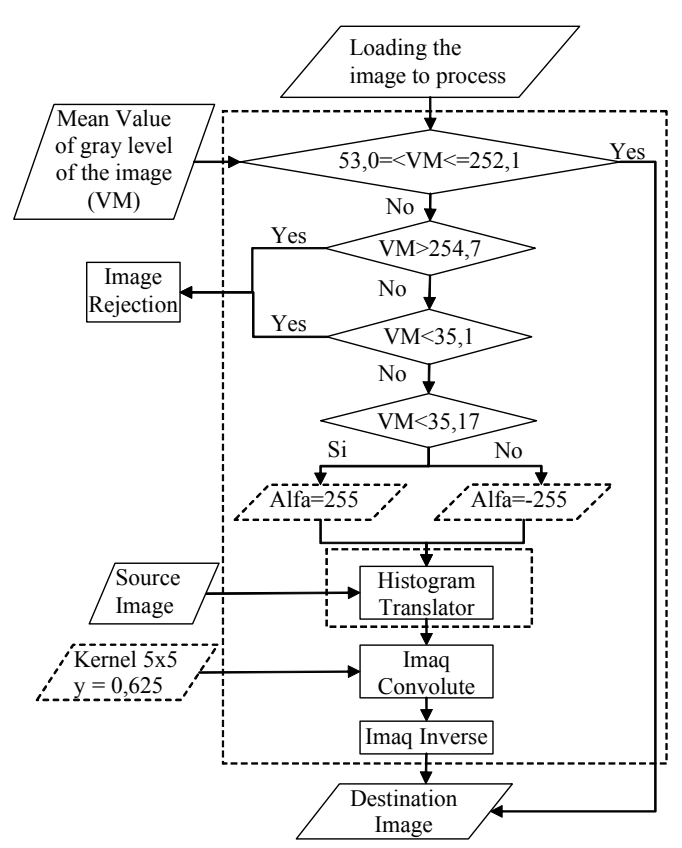

Fig.4 - Block scheme to correct the images affected by the bad exposure alteration.

According to the previous correction procedure, numerical tests show that, in the case of overexposure, the percentage of correct detection is equal to $89,95 \%$. In the contrary, without correction the correct detection 
is $76,38 \%$. In the case of under-exposure the percentage is $85,43 \%$ after the correction and $74,37 \%$ before.

Fig.5 shows the worst overexposed and underexposed images before and after the proposed correction.

The previous correction procedure was compared with the method based on (i) the Histogram Stretching (HS) [11], and (ii) the Equalization of the Histogram (EH) [11], both implemented in LabVIEW environment. The HS consists in stretching the histogram upon all the dynamic range of the image (0255 for 8-bit) and grows up the contrast of the image [5].
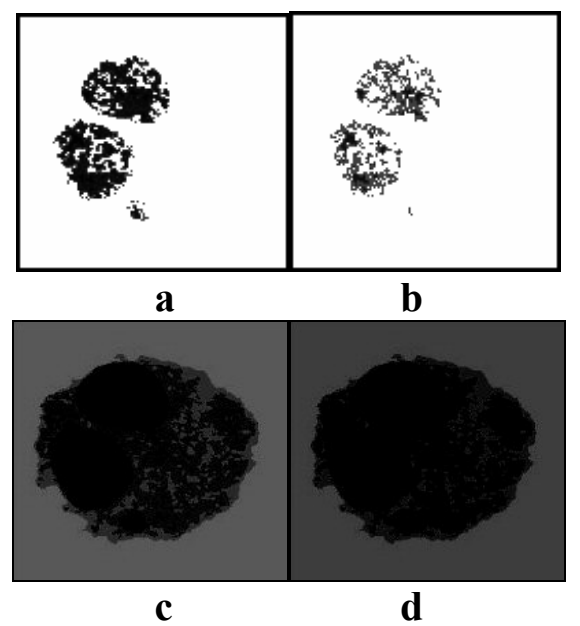

Fig.5 - Worst overexposed image correctly evaluated a) before the correction and b) after the correction; worst underexposed image correctly evaluated c) before the correction and d) after the correction.

The EH consists in altering the gray-level values of the pixels so that they become evenly distributed in the defined grayscale range ( 0 to 255 for 8 -bit). The function associates the equal amount of pixels per constant gray-level interval and takes full advantage of the available shades of gray. This transformation increases the contrast in the images that do not use all gray levels.

In the case of the HS the percentage of correct recognitions is $74,37 \%$, with a decrement of $-2 \%$ for the overexposure, and increment of $2,01 \%$ for the underexposure. In the case of the HE the percentage of correct recognitions is $44,72 \%$, with a decrement of $31,66 \%$ for the overexposure, and increment of $3,01 \%$ for the underexposure.

\section{OUT OF FOCUS ALTERATION}

The definition of the index to evaluate the out of focus alteration arises from the observation that the image affected by this alteration shows lower edge strength in comparison with the sharper one [14] in 3D representation, as shown in Fig. 6.

The valid index is the mean value of the grey levels in the image extracted by the original one by means of the Prewitt's filter [11]. Indeed the filtered sharp image (Fig.7a) is characterized by more bright edges respect to the out of focus image (Fig.7b).

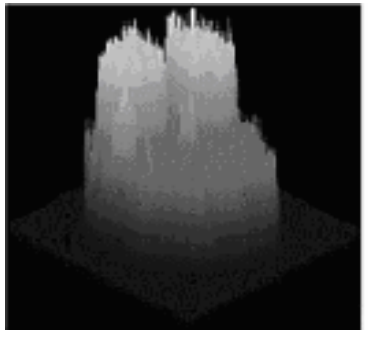

a

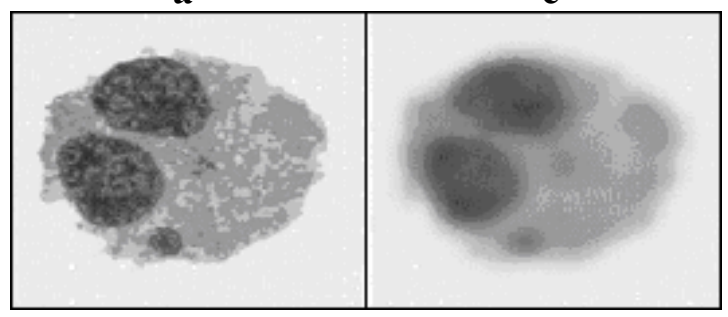

b

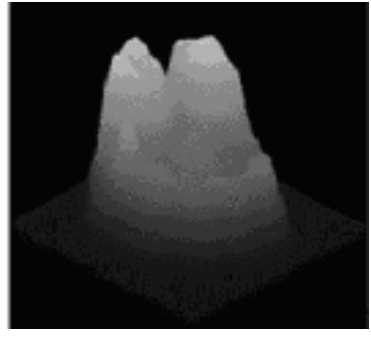

C

d
Fig.6 - a) 3D visualization of the sharp image b), c) 3D visualization of out of focus image d).

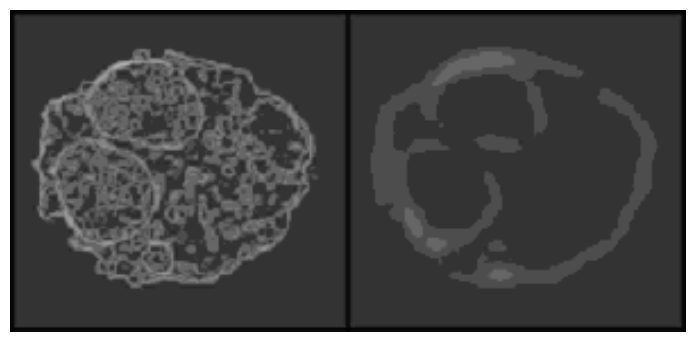

a b

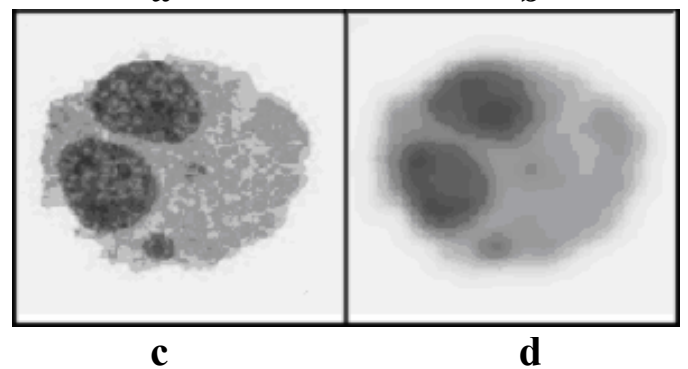

Fig.7 - The images a) and b)are obtained by the application of the Prewitt's filter to c) and d), respectively.

Fig. 8 shows the block scheme to evaluate the index of the out of focus, according to the previous considerations.

The index value belonging to the range [760, 822] denotes that the acquired image must be corrected. Value lower than 760 denotes the image to be rejected. In order to perform the correction and to make the micro nucleuses well distinguished, the image obtained by the application of Laplacian's filter [5] can be added to the original image. The Laplacian filter is used in this case to extract the edges of the image. The kernel describing the Laplacian filter is tuned by try and error technique. It is 
$\begin{array}{lll}-1 & -1 & -1\end{array}$

$-1 \quad 8,5-1$

$-1 \quad-1 \quad-1$

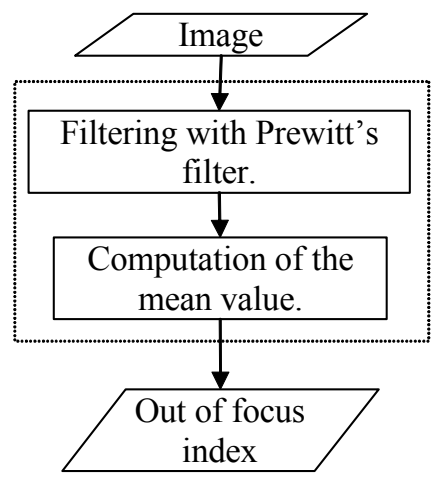

Fig.8 - Block scheme of the method to evaluate the index of the out of focus alteration.

According to the previous correction procedure, numerical tests show that, the percentage of correct detection is equal to $30 \%$. In the contrary, without correction the correct detection is $20 \%$.

Fig.9 shows the block scheme of the method used to correct the out of focus image.

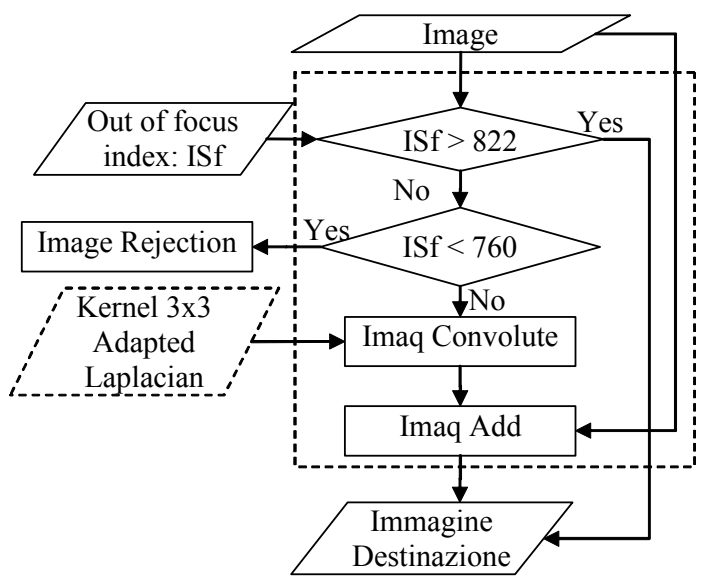

Fig.9 - Block scheme of the method used to correct the images affected by the out of focus alteration.

Fig.10 shows the worst images affected by out of focus correctly evaluated by the pattern matching algorithm a) before and b) after the correction respectively.

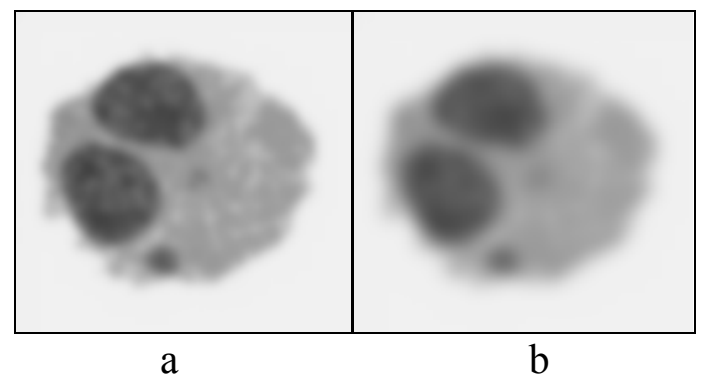

Fig.10 - Worst out of focus image correctly evaluated a) before the correction, and b) after the correction.

\section{THE GAUSSIAN NOISE ALTERATION}

The Gaussian noise alteration is characterize by the presence of pixels with grey value very different from the neighbor pixels.

Fig.11 shows the image affected by different intensity of Gaussian noise and the corresponding 3D representation. It is evident that the number and the entity of the variation in 3D increase with the intensity of the noise.

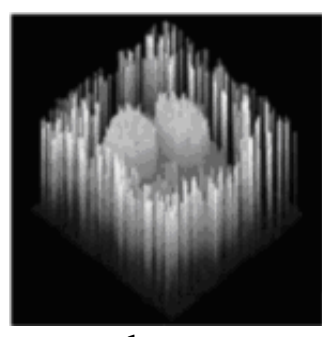

b

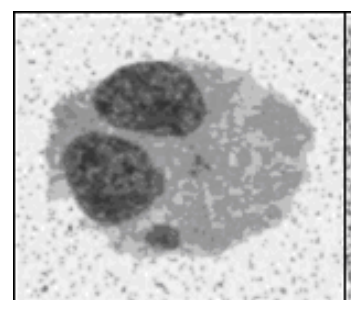

a

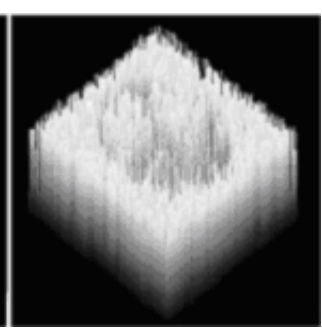

d

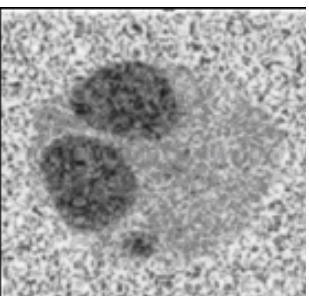

$\mathrm{C}$
Fig.11 - a) Image affected by Gaussian noise and b) corresponding 3D visualization, c) image affected by greater noise intensity than the previous image, and d) corresponding 3D visualization.

Moreover, the Fig $11 \mathrm{~b}$ ) and d) highlight that the pixels belonging to the edges have different gray level respect to part of the pixels of their neighbor. Therefore to measure the noise intensity, it is necessary to distinguish between the information of the image edges and that of the Gaussian noise. A valid index of the Gaussian noise alteration can be obtained following the procedure shown in the block scheme of Fig.12.

In order to evaluate the noise intensity, it is necessary to hypothesize the presences of regions with uniform grey values. This hypothesis is satisfied by all of the examined images. The image is divided into 100 equal dimensional regions. Each one is processed according to the following steps: (i) filtering by highpass filter that furnish information about the quickly variation of the brightness intensity and destroy the information of the background, (ii) evaluation of the absolute value, (iii) computation and storage of the sum of the grey value of each pixel. At the end, the array with 100 values is obtained. The $\mathrm{m}$ lower values correspond to the noise intensity in the uniform regions not affected by the influence of the edges. By averaging the $m$ values the index of the Gaussian noise can be obtained. 


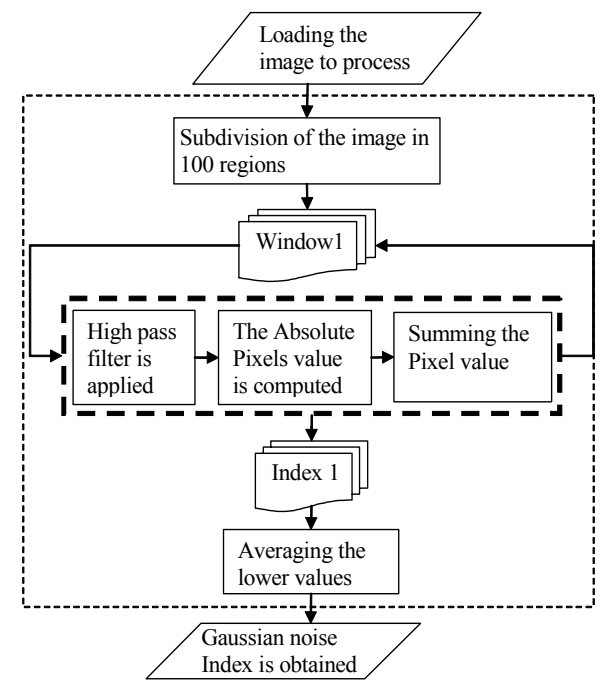

Fig.12 - Block scheme to evaluate the index of the Gaussian noise alteration.

The index value belonging to the range [6238, 13392] denotes that the acquired image must be corrected. Value greater than 13392 denotes the image to be rejected.

In order to perform the correction, numerical tests show that filtering the original image by the Gaussian filter [5] the micro nucleuses can be well distinguished.

The kernel of the Gaussian filter is set by try and error technique. It is :

$\begin{array}{ccccc}\sigma & =0.625 \text { pixels }(5 \times 5) \\ 1 & 2 & 3 & 2 & 1 \\ 2 & 7 & 11 & 7 & 2 \\ 3 & 11 & 17 & 11 & 3 \\ 2 & 7 & 11 & 7 & 2 \\ 1 & 2 & 3 & 2 & 1\end{array}$

The proposed technique to correct the Gaussian noise is shows in Fig 13.

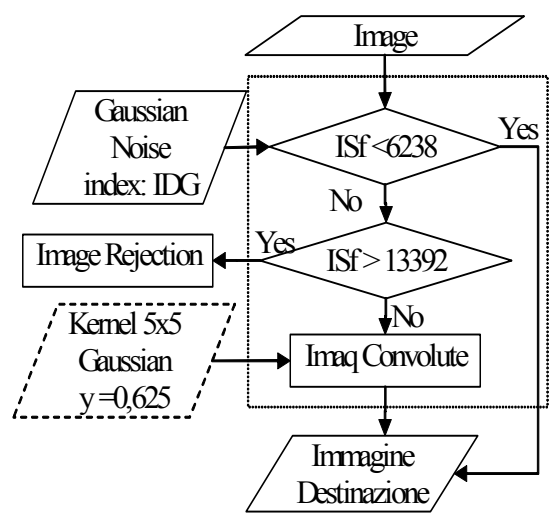

Fig.13 - Block scheme to correct the images affected by the out of focus alteration.

According to the previous correction procedure, numerical tests show that, the percentage of correct detection is equal to $57,58 \% \%$. In the contrary, without the correction the correct detection is $25,25 \%$.

Fig. 14 shows the worst case of the images corrupted by Gaussian noise, correctly analyzed before and after the correction.

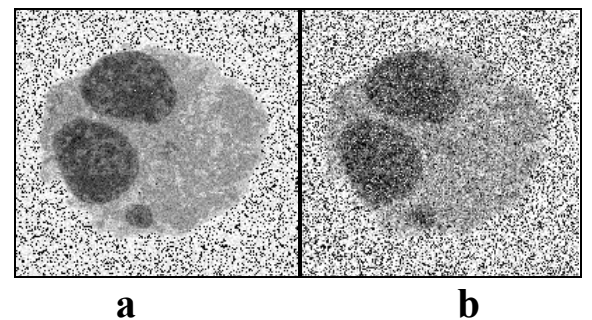

Fig.14 - Worst image affected by Gaussian noise correctly evaluated a) before the correction, and b) after the correction.

\section{CONCLUSIONS}

In the research has been pointed out the procedure to pre-process the image acquired from the new architecture of flow cytometer.

The image pre-processing performs the correction of the alteration arising from (i) the overexposure and the underexposure, (ii) the out of focus, and (iii) the Gaussian noise. The corrected image can be conveniently analyzed by the pattern matching algorithm to detect the micro nucleuses in human lymphocytes. The objectives of the performed correction are (i) the minimization of the doubtful detections, and (ii) the confidence enhancement that the rejected images are not including the micro nucleuses.

Numerical tests highlight the advantages of the performed corrections.

\section{ACKNOWLEDGMENTS}

The Authors whish to thank Prof. Pasquale Daponte of the Faculty of Engineering - University of Sannio, and Prof. Giuseppe Castello of INT-CROP Clinical Immunology Labs - Pascale Institute of Naples, for the help given in all the phases of this work, and the S.Te.Bi Srl, viale delle Acacie 44 - 81031 Aversa (CE) Italy, exclusive producer of the instrument - for its support during the research.

The research is supported by the Italian Ministry of University and Research (MIUR) under the project FIRB RBAU01ZMZ5_002, "Tomography system to acquire 3D images of cells in laminar flow".

\section{REFERENCES}

[1] Z. Darzynkiewicz, Methods in analysis of apoptosis and cell necrosis, Avanced methodologies in flow cytometry, A. Cossarizza, 1997. 
[2] F. Nicoletti, R. Manucci, G. Migliorati, C. Ricciardi, F. Grignani, "Common Methods for measuring apoptotic cell death by flow cytometry", Adv. Method. in flow cytometry, Andrea Cossarizza, 1997.

[3] F. Castello, I. Silvestri, "Linfocita quale dosimetro biologico, Caleidoscopio Italiano $n^{\circ} 130$, Medical Sys., 1999. (Italian).

[4] D. Grimaldi, A. Palumbo, S. Rapuano, "Hardware and software improvements in flow-cytometry measurements", Measurement, vol.36, n.2, September 2004, pp.111-119.

[5] J. C. Russ, The Image Processing Handbook, Fourth Ed., CRC Press, 2002.

[6] R. C. Gonzalez, R. E. Woods, Digital image processing, $2^{\text {nd }}$ Edition, Addison-Wesley, 1992.

[7] G. A. Baxes, Digital Image Processing: Principles and Applications, Paperback, 1994.

[8] P. Camaiti, Guida Ai Ccd per l'astronomia, ed. il Castello, 2000. (Italian).

[9] http://visilab.unime.it/ ianni/slides_CV/cv03.pdf.

[10] C. Hentschel, H. He, "Noise Measurement In Video Images", Philips Research Laboratories, Eindhoven, The Netherlands, Briarcliff Manor NY, USA, 2000.

[11] Imaq Vision Concepts Manual, National Intruments, June, 2003.

[12] Imaq Vision User Manual, National Intruments, May 1999.

[13] Imaq Vision for LabVIEW, User Manual, ,August 2004.

[14] http://zone.ni.com/devzone/devzoneweb.nsf/

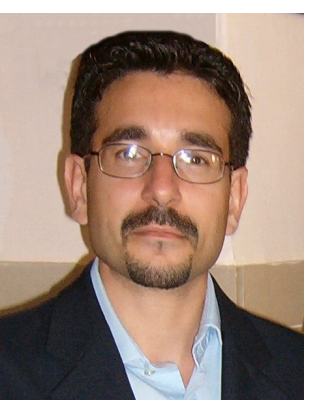

Domenico Luca Carnm, received the M.S. degree in Computer Science Eng. from University of Calabria, Rende, Italy, in the year 2003, where he is currently pursuing the Ph.D. degree in Computer Science. He is developing research work in digital signal processing for measurement in telecommunication systems, characterization of Analog to Digital Converters, image processing for measurement application.

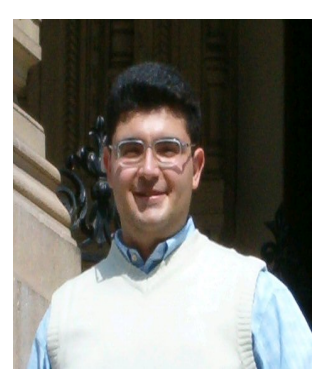

Francesco Lamonaca, was born in Italy. He received the degree in Computer Science Engineering from the University of Calabria in the 2004. He, after working as an independent consultant until 2005, joined the Department of Electronic, Computers and System Science of the
University of Calabria, Italy, as fellow at the Electronic Measurement Laboratory. At the moment it is interested at the researches on the image processing for measurement application.

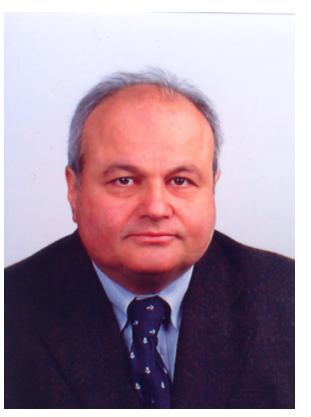

Domenico Grimaldi, was born in Italy. He received the degree "cum laude" in Electrical Engineering from the University of Naples. He, after working as an independent consultant until 1990, joined the Department of Electronic, Computers and System Science of the University of Calabria, Italy, as assistant Professor of Electronic Measurements. At the moment it is Associated Professor of Electronic Measurement at the same Department. Mr. Grimaldi has remained there in a variety of research and management positions. From 1999 it is responsible of the research unit in the frame of National Project PRIN supported by the University Ministry. It was contact person in Tempus Project and Leonardo da Vinci Project of the European Union. Mr. Grimaldi is a member of the IEEE Instrumentation and Measurement Society, and AEI (the Italian Institute of Electrical Engineers). He is coordinator of the IEEE Working Group on "e-tools for Education in Instrumentation \& Measurement". He has published about 140 papers and his current researches include the characterization of measurement transducers, digital signal and image processing for monitoring and testing, virtual instrumentation and distributed measurements, and telecommunication system measurement. 